\title{
IMPROVING GLOBAL NEIGHBORHOOD STRUCTURE MAP DENOISING APPROACH FOR DIGITAL IMAGES
}

\author{
Wasif Shafaet Chowdhury ${ }^{1}$, Jia Uddin ${ }^{2}$, Hamed Alsufyani ${ }^{1}$ and Md. Moinul Hossain ${ }^{1}$ \\ ${ }^{l}$ School of Engineering and Digital Arts, University of Kent, Canterbury, Kent, CT2 7NT \\ ${ }^{2}$ Department of Computer Science \& Engineering, BRAC University, 66 Mohakhali, Dhaka, Bangladesh
}

\begin{abstract}
This paper proposes a new noise reduction model for digital images. In the proposed model, the intensity similarity between the center pixel and its neighboring pixels within a certain window for constructing a Global Neighborhood Structure (GNS) using Dominant Neighborhood Structure (DNS) maps of central pixels has been measured. The intensity similarity was calculated by using the Canberra Distance measurement equation; where the conventional GNS map approach used the Euclidean distance principle. To evaluate the performance of the proposed model, several noise attacks were imposed on two public image datasets and experimental results demonstrated that the proposed model outperforms the conventional GNS map based denoising technique by exhibiting higher PSNR and SNR values.
\end{abstract}

\section{KEYWORDS}

Dominant Neighborhood Structure (DNS), Noise Reduction, Canberra Distance Measurement Equation

\section{INTRODUCTION}

Digital images play a vital role in our daily activities but there is a possibility that the images can get distorted or blurred during the image acquisition process, which is known as noise. These distortion/blurring affects the image pixels, as a result, the original pixel values become inaccurate and escalates the possibility of data loss or can hamper the process of extracting important features from an image. Therefore, noise reduction from digital images is an important factor in the field of image processing (Tin 2011, Chan-yan 2008).

There are several factors that can produce noise in an image such as imperfect instruments, transmission error, compression and etc.(Khellah 2011). These factors result in different types of noises. In this study, five types of noises such as Gaussian Noise, Localvar Noise, Salt and Pepper Noise, Poison Noise and Speckle Noise have been imposed on the tested image datasets. The Gaussian Noise is a randomly generated noise values which affects the image pixels. In a Gaussian Noise added image both the true pixel values and random Gaussian noise added values exists. It distributes the noise values by using the Gaussian probability density function but it is free from the intensity of an image pixel (Hoshyar et al 2014, Luisier et al 2011). On the other hand, the Localvar Noise is basically a zero-mean Gaussian white noise. The difference lies in the intensity, the Localvar noise is dependent on the pixel intensity of an image (Tin 2011). In addition to that, Salt and Pepper is another type of noise which is the combination of black and white spots in an image. It can occur in an image during the data transmission process because of errors in the image sensors (Hoshyar et al 2014). Furthermore, Poison Noise also known as Photon noise is dependent on measurement of light, photon direction and quantized nature of light. Speckle noise is represented on the image by random values which are multiplied by the pixel values of that image. In medical literature, this noise is known as texture (Unser 1995, Rabbani et al 2008).

During the last decades, noise reduction has become an important field of research and it is considered as one of the most significant parts of signal processing (Hazavei and Shahdoosti 2017). Various denoising approaches, (known as filters), have been developed and many of them have shown very impressive results. Filters can be divided into Linear and Non-Linear Filters. These filters are usually selected based on the nature and the type of data (Cadena et al 2017). Filters like Linear, Median, Gaussian Smoothing and Nonlocal Means are commonly used and well discussed in the literature of (Hazavei and Shahdoosti 2017, 
Hoshyar et al 2014, Cadena et al 2017, Khellah 2014). A brief discussion of some of the popular filters has been presented in this work.

One of the most commonly used filters in image processing is the Linear Filter. The mechanism of this filter is to take an average of the pixels that are situated within a local region. The number of pixels included in the local region is specified by a kernel which can be different in size. It has shown adequate results in case of removing the uniform and Gaussian noise. However, the limitations of this method are blurring and smoothen the edges of an image. In addition, the dominance of the blurring is proportional to the size of the kernel. The bigger the kernel is the predominant the blurring becomes (Hazavei and Shahdoosti 2017, Cadena et al 2017).

In the context of noise reduction, median filtering is widely used nonlinear denoising approach, because of the preservation process of edges while removing noise (Hazavei and Shahdoosti 2017). The basic idea of this approach is to replace the center of a window with the median, which is achieved by sorting the pixel values of the window numerically and picking up the center. It is suggested that the window size should be an odd number because it is easier to find the centre value from the sorted numbers. The window slides pixel by pixel through the entire image. It is clear that this procedure is applicable and practical for removing the Speckle and Salt and Pepper noises (Cadena et al 2017, Castro and Donoho 2009, Hsieh et al 2013). However, the time complexity is high because of calculating the median of the kernel and process each and every pixel of an image. Additionally, similar to Linear Filter this algorithm blurs the input image even at low densities (Cadena et al 2017, Ben 2006, Gonzalez and Woods 2008).

Gaussian Filter is a 2-D convolution operation where each pixel of the image is replaced by a weighted average of its neighbors (Hazavei and Shahdoosti 2017, Mengqi 2011). This filter is similar to the Linear Filter but the kernel is different (Cadena et al 2017). In Gaussian Filter, the convolution operation is based on a Gaussian function which provides an estimation of transformation to be applied at each pixel in an image. The representation of the image is also similar to a collection of discrete values. Therefore, it is essential to produce a discrete approximation to the function before applying the convolution (Pascal 2013). This filtering approach is considered an efficient method but it has some drawbacks such as for noises that are subject to a normal distribution (Wang et al 2014). It is particularly known for blurring and suppressing the noise (Pei et al 2007). It also misrepresents the signal, dislocates the edges or vanishes them (Lu and Jain 1989, Clark 1989). As a result, there is a possibility of image distortion or failure to achieve the desired output.

Khellah (2014) reported a nonlocal means filtering approach which produced very impressive outcomes in case of reducing noise from images. This filter is based on an idea that natural and textured images have redundancy and it is not necessary that pixels with similar values should be located in a nearby spatial neighborhood. It uses local neighbourhoods to calculate the similarity of any two given pixels. Additionally, it scans the entire image to calculate the differences between the corresponding neighborhoods for each pixel being processed. Whereas, in windowed nonlocal means filter, the computation is limited within a search window where the processed pixel is at the center (Buades et al 2005). The major drawback of this filter is that if noise level increases, the similarity selection also increases. This limitation leads to blurring or strong smoothing. Because, at high noise levels, the averaged intensity values of the pixels in the search window is nearly equal to the restored pixels. The technique proposed by Khellah (2014) addressed the drawback of the windowed nonlocal means filter by using an algorithm that was developed in (Khellah 2011). DNS map was used to determine the most similar pixels surrounding any given pixel within a search window. While filtering a given pixel, the DNS map was applied to exclude any insignificant pixel from the restoration of a given pixel (Khellah 2014). The extracted global image features were then classified using the DNS map (Khellah 2011). Exploiting the high redundancy that exists in texture images in general and specifically inhomogeneous texture images with repetitive patterns are the basis of the DNS map. The DNS map was applied by determining the most similar pixels surrounding any given pixel within a searching window.

This paper presents an improved DNS algorithm for image noise reduction. The improved algorithm has been evaluated by applying different distance measurement principles. The detail implementation of this algorithm has been described and verified through PSNR and SNR. A comparative study has also been carried out to investigate the optimal window sizes and the equation under different datasets and noises. The results obtained from this study have been presented and analysed. The result demonstrates that the proposed algorithm offers the best noise reduction from images in comparison to the conventional DNS algorithm. 


\section{PROPOSED MODEL}

Figure 1 shows the block diagram of the proposed Global Neighborhood Structure (GNS) map using the Dominant Neighborhood Structure (DNS) map. A detail description of the proposed model has been given below.

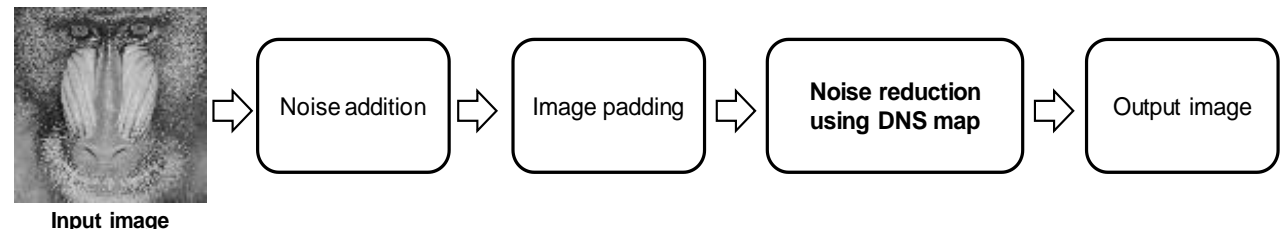

Figure 1. Overview of the proposed noise reduction technique

\subsection{Noise Addition}

The initial step was to convert the image from RGB to gray scale. The reason was that gray scale based processing techniques provide advantages in case of implementing mathematical equations as the pixel values range between $0-255$. After the RGB to gray scale conversion, noise has been added on the gray scale image. Figure 2 shows five different noise levels added to the input image. Once the noise addition was completed, the image was padded and the then forwarded to the next step of the algorithm. The proposed DNS algorithm was applied to reduce the noise and their results were analyzed. Similar approach was considered for the rest of the noises.

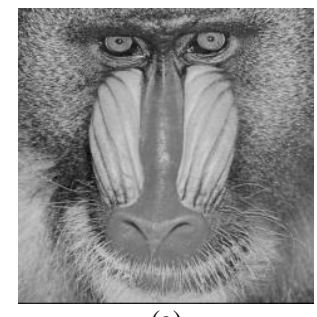

(a)

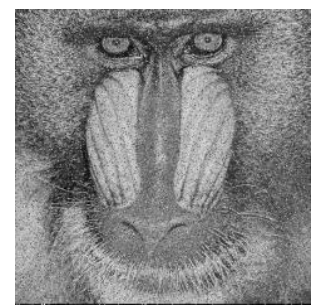

(d)

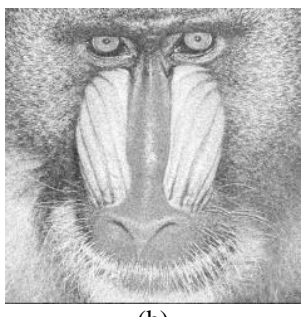

(b)

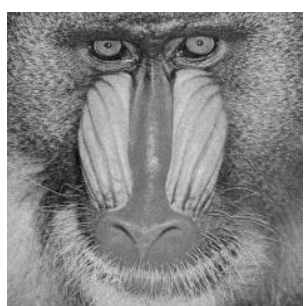

(e)

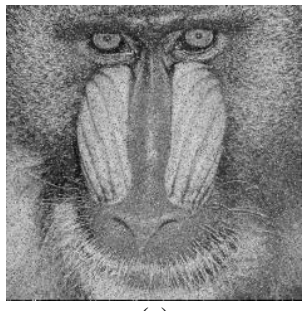

(c)

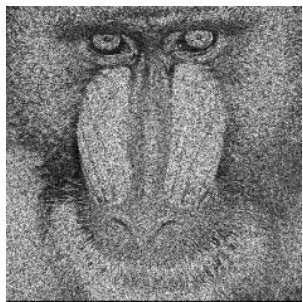

(f)

Figure 2. Example of different noise added images: a) Input image, b) Gaussian, c) Salt and Pepper, d) Localvar,

e) Poison and f) Speckle

\subsection{Image Padding}

In this stage, the border of the noise added image was extended. The reason behind extending the border was that at the beginning of the sliding process, the searching window could have ignored the pixels that were situated at the edge of the image in order to provide space to the neighbor window. As a result, the accuracy of the DNS algorithm would have degraded. As depicted in Figure 3, the image was padded by duplicating the pixel values from the upper and lowermost rows and from the left and rightmost columns. 


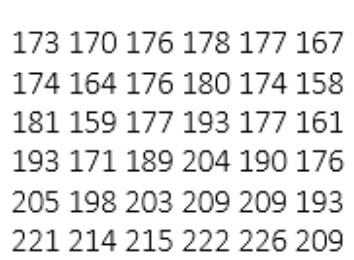

173173170176178177167167

173173170176178177167167

174174164176180174158158

181181159177193177161161

193193171189204190176176

205205198203209209193193

221221214215222226209209

221221214215222226209209

Figure 3. Sample representation of the image padding approach

\subsection{Noise Reduction using DNS Map}

As described in (Khellah 2014, Uddin et al 2014), there are two windows that should be built to construct the DNS map. The first one is the searching window and the second one is the neighborhood window. The searching window slides the entire image and at the same time, the neighborhood window scans the searching window. The sliding process of the searching window starts from the first pixel of the padded image. During the scanning process, the differences of pixel values between the neighborhood and the central pixel window were calculated by using the Canberra equation (6). Here, the result of Canberra replaces the central pixel of the neighborhood window. It is clear that the neighborhood window does not scan the central pixel window while going through the searching window. The reason is the difference in pixel value between these two windows would always be zero. Figure 4 illustrates a graphical representation of the proposed DNS map.

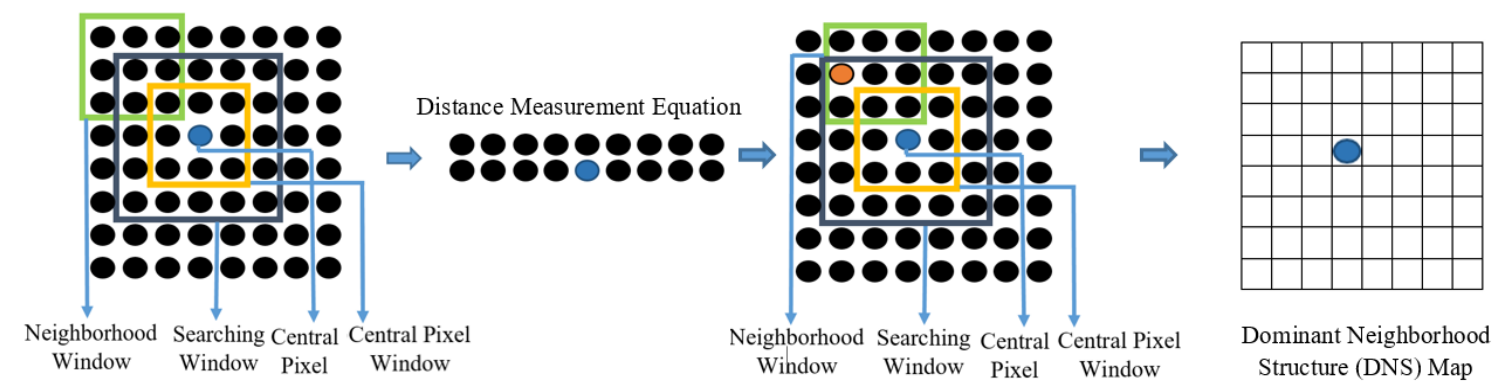

Figure 4. Graphical representation of the DNS map

The size of the searching window, neighborhood window and the gap between the central pixels are the three important factors that can affect the result of the proposed denoising approach (Uddin and Kim 2014). Therefore, the sizes have been calculated by adopting a try and error procedure. The outcome of the try and error process exhibited that the optimal size for the searching and the neighborhood windows were $21 \times 21$ and 19x19 respectively. As the size of the neighborhood window was 19x19, the size of the central pixel window was set to 19x19. An analysis of the window size determination has been discussed in Section 3 .

Six distance measurement equations were taken under consideration to determine the optimal one. Table 1 shows the list of the selected equations. In these equations $d(x, y)$ represents the central pixel of the neighborhood window and $x_{i}$ represents the pixels within the neighborhood window and $y_{i}$ represents the pixels located in the central pixel window. According to the experimental results of this study, the Canberra Distance equation provides the best results for the denoising approach. A detail explanation of the outcomes of these distance measurement equations has been presented in Section 3. 
Table 1. List of distance measurement equations

\begin{tabular}{l|c}
\hline Equation Name & Equation \\
\hline Euclidean Distance & $d(x, y)=\sqrt{\sum x_{i}-y_{i}}$ \\
Squared Euclidean distance & $d(x, y)=\sum\left(x_{i}-y_{i}\right)^{2}$ \\
Manhattan Distance & $d(x, y)=\sum\left|\left(x_{i}-y_{i}\right)\right|$ \\
Bary Curtis Distance & $d(x, y)=\frac{\sum\left|x_{i}-y_{i}\right|}{\sum x_{i}+y_{i}}$ \\
Cosine Correlation Distance & $d(x, y)=\frac{\sqrt{\sum x_{i} \times y_{i}}}{\sqrt{\sum\left(x_{i}\right)^{2} \sum\left(y_{i}\right)^{2}}}$ \\
\hline Canberra Distance (Proposed scheme) & $d(x, y)=\frac{\sum\left|x_{i}-y_{i}\right|}{\sum\left|x_{i}+y_{i}\right|}$ \\
\hline
\end{tabular}

\subsection{Dataset}

In order to analyse the efficiency of the proposed model two public datasets have been used in this study. The first one was the 'Face Recognition ORL Database' (Petitcolas access 2019) and the second dataset is the texture images from (Brodatz 1966). Figure 5 shows some sample texture images that have been used in this study.

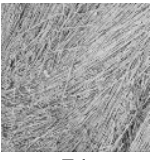

I1

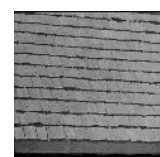

I9

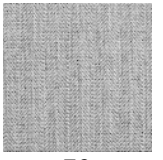

$\mathbf{I 2}$

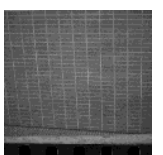

I10

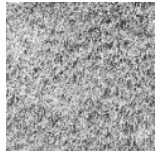

I3

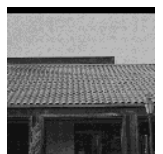

I11

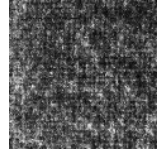

14

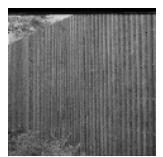

I12

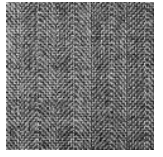

I5

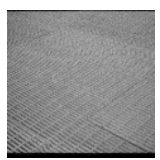

I13

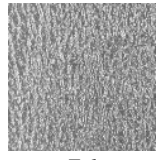

I6

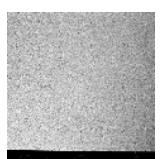

I14

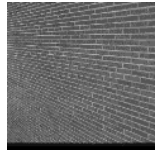

I7

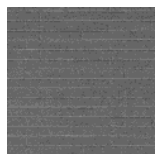

I15

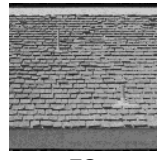

I8

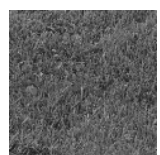

I16

Figure 5. Sample texture images used in this experiment

\section{RESULTS AND DISCUSSIONS}

The results analysis has been divided as follows. Firstly, while calculating the distance between the pixels within the neighborhood and central pixel window, six distance measurement equations (1) - (6) have been implemented, where the equation (1) is the traditional one. A comparative study has been carried out to investigate the best equation based on higher PSNR and SNR results. Secondly, the try and error procedure has been undertaken to determine the optimal window sizes for both the searching window (SW) and the neighborhood window (NW). Table 2 demonstrates the outcomes of the six distance measurement equations for the try and error approach on Speckle noisy image.

\subsection{Determining the Optimal Equation}

From Table 2, it can be seen that for the first three equations (Euclidean, Squared Euclidean and Manhattan) the PSNR values fluctuates within the range of 47.05 to 54.39 with respect to the changes in window sizes. 
Another noticeable point is that the PSNR results of equation (2) and (3) are similar to the PSNR results of equation (1) for most of the cases. It evident that equations (2) and (3) did not provide any significant changes while measuring the intensity similarity for DNS map. Looking more closely, the PSNR values for the last three principals (Bary Curtis, Canberra and Cosine Correlation), ranges between 49.53 to 62.99, which are significantly higher than the first three equations. Therefore, equation (4), (5) or (6) are the candidate equations, to be the optimal ones for the DNS map. Additionally, the PSNR results that were obtained for the proposed equation (Equation 6) was higher than equations (4) and (5) and the Euclidean equation as well, for all sizes of SW and NW. Moreover, as the window sizes increase the results of Equation (6) also increases and get noticeably higher in comparison with all the other equations. Hence, the Canberra equation (Equation (6)) has been selected as the optimal equation in order to measure the intensity similarity between the center pixel and its neighboring pixels within a certain window for the DNS map based GNS algorithm.

Table 2. PSNR results of distance measurements against different window size

\begin{tabular}{|c|c|c|c|c|c|c|}
\hline $\begin{array}{c}\text { Window Sizes } \\
\text { SW x NW }\end{array}$ & Euclidean & $\begin{array}{c}\text { Squared } \\
\text { Euclidean }\end{array}$ & Manhattan & Bary Curtis & $\begin{array}{c}\text { Cosine } \\
\text { Correlation }\end{array}$ & $\begin{array}{c}\text { Proposed } \\
\text { (Canberra) }\end{array}$ \\
\hline $5 \times 5 \& 3 \times 3$ & 54.39 & 47.05 & 47.05 & 56.80 & 56.89 & 57.18 \\
\hline $7 \times 7 \& 5 \times 5$ & 49.29 & 48.78 & 48.78 & 54.53 & 54.63 & 55.27 \\
\hline $9 \times 9 \& 7 x 7$ & 48.75 & 48.75 & 48.75 & 52.72 & 52.80 & 53.71 \\
\hline $11 \times 11 \& 9 \times 9$ & 49.31 & 49.31 & 49.31 & 52.26 & 52.34 & 53.49 \\
\hline $13 \times 13 \& 11 \times 11$ & 48.52 & 48.52 & 48.52 & 53.35 & 53.44 & 55.59 \\
\hline $15 \times 15 \& 13 \times 13$ & 48.26 & 48.26 & 48.26 & 54.09 & 54.19 & 56.92 \\
\hline $17 \times 17 \& 15 \times 15$ & 47.81 & 47.81 & 47.81 & 55.10 & 55.21 & 59.68 \\
\hline $19 \times 19 \& 17 \times 17$ & 48.85 & 48.85 & 48.85 & 52.99 & 53.08 & 57.05 \\
\hline $21 \times 21 \& 19 \times 19$ & 47.83 & 47.83 & 47.83 & 55.07 & 55.18 & 62.99 \\
\hline $23 \times 23 \& 21 \times 21$ & 48.50 & 48.50 & 48.50 & 53.36 & 53.45 & 58.95 \\
\hline $25 \times 25 \& 23 \times 23$ & 49.05 & 49.05 & 49.05 & 52.75 & 52.83 & 61.75 \\
\hline $27 \times 27 \& 25 \times 25$ & 49.55 & 49.55 & 49.55 & 51.79 & 51.87 & 59.27 \\
\hline $29 \times 29 \& 27 \times 27$ & 49.56 & 49.56 & 49.56 & 51.87 & 51.95 & 59.47 \\
\hline $31 \times 31 \& 29 \times 29$ & 49.88 & 49.88 & 49.88 & 51.60 & 51.67 & 61.22 \\
\hline $33 \times 33$ \& 31x31 & 51.84 & 51.84 & 51.84 & 49.63 & 49.69 & 57.69 \\
\hline $35 \times 35 \& 33 \times 33$ & 51.58 & 51.58 & 51.58 & 49.53 & 49.59 & 57.22 \\
\hline $37 \times 37 \& 35 \times 35$ & 50.64 & 50.64 & 50.64 & 50.65 & 50.72 & 55.82 \\
\hline
\end{tabular}

\subsection{Optimal Window Size Determination}

In order to determine the optimal window sizes, a try and error approach has been carried out. Initially, the window sizes of SW \& NW were set to SW 5x5 \& NW 3x3 and then they were increased gradually by adding two pixels in both the windows. Table 2 demonstrates the gradual increment of the window sizes and represents their corresponding PSNR results. The peak PSNR value of 62.99 was observed for SW $21 \times 21$ \& NW 19x19. Therefore, these window sizes have been considered as the optimal sizes for the proposed model. Note that, the results presented in Table 2 were obtained from the outcomes of the proposed model on a Speckle Noise added image.

As five types of noises were imposed on the tested dataset images. Therefore, further analysis of the proposed algorithm has been done for the other types of noises. Table 3 demonstrates the results of the traditional equation (Euclidean equation) and the proposed equation (Canberra equation). From the table (Table 3) it is clear that the proposed model of the DNS map exhibits better results in comparison with the traditional algorithm for all types of noises based on PSNR and SNR results.

Table 3. Comparison between proposed and Euclidean equation

\begin{tabular}{|c|c|c|c|c|c|c|}
\hline & Noise & Gaussian & Salt and Pepper & Localvar & Poisson & Speckle \\
\hline \multirow{2}{*}{ PSNR } & Euclidean & 48.02 & 48.81 & 47.23 & 48.24 & 47.83 \\
\cline { 2 - 7 } & Canberra & 55.76 & 54.55 & 54.16 & 55.42 & 62.99 \\
\hline \multirow{2}{*}{ SNR } & Euclidean & 42.65 & 43.44 & 42.11 & 42.80 & 42.49 \\
\cline { 2 - 7 } & Canberra & 50.39 & 49.18 & 49.04 & 49.97 & 57.65 \\
\hline
\end{tabular}


Further to validate the proposed algorithm, the Canberra (Can) and Euclidean (Euc) were explored for 16 different texture images as shown in Figure 5. The five different noises were applied to the texture images. The optimal window sizes of SW 21x21 \& NW 19x19 was used to calculate the PSNR and their results have been presented in Table 4. It is clear that the proposed scheme provides better performance compared to the traditional equation. For example, a significant difference can be seen for the images I3, I4, I5, I6, I7, I9, I10, I11, I12, I13, I14, I15 and I16, and almost $82 \%$ improvement was achieved by the proposed model. Hence, it is suggested that the Canberra principal can be used to measure the intensity similarity between the center pixel and its neighboring pixels within a certain window for constructing the DNS map instead of Euclidean equation.

Table 4. A PSNR based comparison between the Euclidean and Canberra equations on texture images

\begin{tabular}{|c|c|c|c|c|c|c|c|c|c|c|c|c|c|}
\hline Img & Equ & Gau & S\&P & Loc & Poi & Spe & Img & Equ & Gau & S\&P & Loc & Poi & Spe \\
\hline \multirow{2}{*}{ I1 } & Euc & 50.37 & 49.17 & 50.20 & 50.21 & 50.14 & \multirow{2}{*}{19} & Euc & 46.62 & 46.25 & 46.93 & 46.40 & 46.16 \\
\hline & Can & 50.79 & 51.25 & 49.90 & 50.55 & 50.08 & & Can & 57.05 & 59.12 & 56.00 & 58.30 & 58.99 \\
\hline \multirow{2}{*}{ I2 } & Euc & 49.64 & 49.68 & 50.11 & 50.87 & 50.85 & \multirow{2}{*}{ I10 } & Euc & 45.60 & 45.39 & 45.28 & 46.81 & 47.03 \\
\hline & Can & 51.74 & 51.00 & 49.73 & 50.47 & 49.97 & & Can & 64.31 & 67.07 & 64.33 & 68.24 & 68.10 \\
\hline \multirow{2}{*}{ I3 } & Euc & 49.73 & 50.55 & 49.91 & 49.91 & 50.20 & \multirow{2}{*}{ I11 } & Euc & 47.38 & 47.52 & 49.92 & 47.55 & 47.53 \\
\hline & Can & 53.31 & 52.54 & 53.92 & 52.92 & 52.84 & & Can & 54.26 & 61.51 & 50.45 & 61.67 & 63.43 \\
\hline \multirow{2}{*}{ I4 } & Euc & 47.30 & 47.54 & 47.11 & 47.08 & 46.98 & \multirow{2}{*}{ I12 } & Euc & 48.04 & 48.03 & 48.59 & 47.96 & 47.51 \\
\hline & Can & $\begin{array}{l}59.41 \\
\end{array}$ & \begin{tabular}{|l|l|}
55.83 \\
\end{tabular} & $\begin{array}{l}55.43 \\
\end{array}$ & 65.95 & 63.74 & & Can & 62.14 & $\begin{array}{l}57.72 \\
\end{array}$ & 52.74 & 58.68 & 62.67 \\
\hline \multirow{2}{*}{ I5 } & Euc & 50.82 & 50.12 & 52.37 & 50.27 & 49.93 & \multirow{2}{*}{ I13 } & Euc & 45.63 & 45.62 & 46.65 & 45.52 & 45.46 \\
\hline & Can & 52.41 & 52.10 & 48.96 & 53.37 & $\begin{array}{l}55.82 \\
\end{array}$ & & Can & 63.92 & 64.53 & 55.83 & 65.55 & 66.39 \\
\hline \multirow{2}{*}{ I6 } & Euc & 50.46 & 50.50 & 48.24 & 50.65 & 50.37 & \multirow{2}{*}{ I14 } & Euc & 45.14 & 45.04 & 46.01 & 46.79 & 47.90 \\
\hline & Can & 53.71 & 52.99 & 57.32 & 52.37 & $\begin{array}{l}53.41 \\
\end{array}$ & & Can & 67.57 & $\begin{array}{l}56.89 \\
\end{array}$ & 57.43 & 54.25 & 58.42 \\
\hline \multirow{2}{*}{ I7 } & Euc & 45.72 & 45.35 & 45.98 & 47.34 & 47.20 & \multirow{2}{*}{ I15 } & Euc & 50.06 & 49.56 & 48.21 & 49.95 & 49.92 \\
\hline & Can & 61.21 & 67.15 & 60.09 & 65.34 & $\begin{array}{l}65.62 \\
\end{array}$ & & Can & $\begin{array}{l}54.58 \\
\end{array}$ & $\begin{array}{l}53.78 \\
\end{array}$ & 57.74 & $\begin{array}{l}53.35 \\
\end{array}$ & 55.20 \\
\hline \multirow{2}{*}{ I8 } & Euc & 50.50 & 50.17 & 50.73 & 49.91 & 50.42 & \multirow{2}{*}{ I16 } & Euc & 47.92 & 48.88 & 48.20 & 48.84 & 48.50 \\
\hline & Can & 50.71 & 51.39 & 49.37 & 51.66 & 50.71 & & Can & $\begin{array}{r}63.58 \\
\end{array}$ & 55.79 & 55.40 & 56.24 & 59.15 \\
\hline
\end{tabular}

\section{CONCLUSION}

A noise reduction model for the digital image has been developed and implemented by constructing a GNS map based DNS algorithm. Various distance measurement equations and noises were used to verify the model. Their PSNR and SNR values were calculated. Optimal window sizes for the proposed model was also been investigated. The difference between the central window pixels and the neighbourhood window pixels were calculated by using the Canberra distance measurement technique. A comparative study has been carried out to further verify the proposed algorithm. The results obtained from the comparative study demonstrates that the proposed model is capable of reducing the noise $\approx 82 \%$ for texture images in comparison to the conventional DNS algorithm.

\section{REFERENCES}

Ben, W., 2006. Fast median and bilateral filtering. ACM SIGGRAPH 2006 Papers, pp. 519-526.

Brodatz, P., 1966. Texture: A Photographic Album for Artists and Designers, Dover, New York.

Buades, A. et al, 2005. A non-local algorithm for image denoising. Proceedings of the International Conference on Computer Vision and Pattern Recognition, pp. 60-65.

Cadena, L. et al, 2017. Noise reduction techniques for processing of medical images. Proceedings of the World Congress on Engineering 2017, London UK, Vol. I, pp. 496-500.

Castro, E. A. and Donoho, D. L., 2009. Does median filtering truly preserve edges better than linear filtering?. The Annals of Statistics, pp. 1172-1206. 
Chang-yan, C. et al, 2008. Study on methods of noise reduction in a stripped image. The International Archives of the Photogrammetry, Remote Sensing and Spatial Information Sciences, China, Vol. XXXVII, pp. 213 - 216.

Clark, J. J., 1989. Authenticating edges produced by zero-crossing algorithms. IEEE Transactions on Pattern Analysis and Machine Intelligence, Vol. PAMI-1 1, pp. 43-57.

Gonzalez, R. C. and Woods, R. E., 2008. Digital Image Processing. Prentice Hall, Upper Saddle River, New Jersey, USA, ch. 5.

Hazavei, S. M. and Shahdoosti, H. R., 2017. A new method for removing the moire' pattern from images. CoRR, Vol. abs/ 1701.09037, pp. 1-6.

Hoshyar, A. N. et al, 2014. Comparing the performance of various filters on skin cancer images. International Conference on Robot PRIDE, Vol. 42, pp. 32-37.

Hsieh, M. H. et al, 2013. Fast and efficient median filter for removing 1-99\% levels of salt-and-pepper noise in images. Engineering Applications of Artificial Intelligence, Vol. 26, pp. 1333-1338.

Khellah, F. M., 2011. Texture classification using dominant neighborhood structure. IEEE Transactions on Image Processing, Vol. 20, No. 11, pp. 3270-3279.

Khellah, F. M., 2014. Textured image denoising using dominant neighborhood structure. Arabian Journal for Science and Engineering, Vol. 39, No. 5, pp. 3759-3770.

Lu, Y. and Jain, R. C., 1989. Behavior of edges in scale space. IEEE Transactions on Pattern Analysis and Machine Intelligence, Vol. PAMI-I 1, pp. 337-356.

Luisier, F. et al, 2011. Image denoising in mixed Poison-Gaussian noise. IEEE Transaction on Image Processing, Vol. 20, No. 3, pp. 696-708.

Mengqi, L., 2011. Research on Image De-Noising Enhancement. Savonia University of Applied Sciences, Bachelor's thesis.

Petitcolas, F. a. p. (n.d.). Face recognition ORL database. Retrieved April 29, 2019, from https://homepages.cae.wisc.edu/ ece533/images/

Pascal, G., 2013. A Survey of Gaussian Convolution Algorithms: Main Page. (2019). Retrieved from http://dev.ipol.im/ getreuer/code/doc/gaussian_20131215_doc/.

Pei, Y. H. et al, 2007. Generic 2D Gaussian smoothing filter for noisy image processing. IEEE Region 10 Conference (TENCON), pp. 1-4.

Rabbani, H. et al, 2008. Speckle noise reduction of medical ultrasound images in complex wavelet domain using mixture priors. IEEE Transactions on Biomedical Engineering, Vol. 55, No. 9, pp. 2152-2160.

Tin, H. H. K., 2011. Removal of noise by median filtering in image processing. The 6th Parallel and Soft Computing (PSC), pp. 1-3.

Uddin, J. and Kim, J. M., 2014. A Reliable Fault Detection and Classification Model of Induction Motors using Texture Features and Multi-class Support Vector Machines. The 2014 FTRA International Workshop on Advanced Multimedia Computing (AMC - 14) Proceedings, pp. 1- 6.

Uddin, J. et al, 2014. Accelerating 2D Fault Diagnosis of an Induction Motor using a Graphics Processing Unit. International Journal of Multimedia and Ubiquitous Engineering, Vol. 9, No. 7, pp. 1-13.

Unser, M., 1995. Texture classification and segmentation using wavelet frames. IEEE Transactions on Image Processing, Vol. 4 (11), pp.1549-1560.

Wang, M. et al, 2014. A new image denoising method based on Gaussian filter. International Conference on Information Science, Electronics and Electrical Engineering (ISEEE), Japan, pp. 163-167. 